\title{
Effects of cytokine blocking agents on hospital mortality in patients admitted to ICU with acute respiratory distress syndrome by SARS-CoV-2 infection: retrospective cohort study
}

\author{
Irene Coloretti, ${ }^{1}$ Stefano Busani, ${ }^{1}$ Emanuela Biagioni, ${ }^{1}$ Sophie Venturelli, ${ }^{1}$ Elena Munari, ${ }^{1}$ Marco Sita, ${ }^{1}$ \\ Lorenzo Dall'Ara, ${ }^{1}$ Martina Tosi, ${ }^{1}$ Enrico Clini, ${ }^{2}$ Roberto Tonelli, ${ }^{2}$ Riccardo Fantini, ${ }^{2}$ Cristina Mussini, ${ }^{3}$ \\ Marianna Meschiari, ${ }^{3}$ Giovanni Guaraldi, ${ }^{3}$ Andrea Cossarizza, ${ }^{4}$ Gaetano Alfano, ${ }^{5}$ Massimo Girardis, \\ for the Modena COVID-19 Working Group (MoCo19)* \\ ${ }^{1}$ Intensive Care Unit, University Hospital of Modena \\ ${ }^{2}$ Respiratory Disease Unit, University Hospital of Modena \\ ${ }^{3}$ Infectious Disease Unit, University Hospital of Modena \\ ${ }^{4}$ Immunology Laboratory, University of Modena and Reggio Emilia \\ ${ }^{5}$ Nephrology Dialysis and Transplant Unit, University Hospital of Modena, Italy
}

\begin{abstract}
Background: The use of cytokine-blocking agents has been proposed to modulate the inflammatory response in patients with COVID-19. Tocilizumab and anakinra were included in the local protocol as an optional treatment in critically ill patients with acute respiratory distress syndrome (ARDS) by SARS-CoV-2 infection. This cohort study evaluated the effects of therapy with cytokine blocking agents on in-hospital mortality in COVID-19 patients requiring mechanical ventilation and admitted to intensive care unit.

Methods: The association between therapy with tocilizumab or anakinra and in-hospital mortality was assessed in consecutive adult COVID-19 patients admitted to our ICU with moderate to severe ARDS. The association was evaluated by comparing patients who received to those who did not receive tocilizumab or anakinra and by using different multivariable Cox models adjusted for variables related to poor outcome, for the propensity to be treated with tocilizumab or anakinra and after patient matching.

Results: Sixty-six patients who received immunotherapy (49 tocilizumab, 17 anakinra) and 28 patients who did not receive immunotherapy were included. The in-hospital crude mortality was 30,3\% in treated patients and $50 \%$ in nontreated (OR 0.77, 95\% CI 0.56-1.05, $\mathrm{p}=0.069)$. The adjusted Cox model showed an association between therapy with immunotherapy and in-hospital mortality (HR 0.40, 95\% CI 0.19-0.83, p=0.015). This protective effect was further confirmed in the analysis adjusted for propensity score, in the propensity-matched cohort and in the cohort of patients with invasive mechanical ventilation within 2 hours after ICU admission.

Conclusions: Although important limitations, our study showed that cytokine-blocking agents seem to be safe and to improve survival in COVID-19 patients admitted to ICU with ARDS and the need for mechanical ventilation.
\end{abstract}

Key words: COVID-19; acute respiratory distress syndrome; tocilizumab; anakinra; intensive care unit; mechanical ventilation.

Correspondence: Coloretti Irene, Intensive Care Unit, Department of Anaesthesiology and Intensive Care, University Hospital of Modena, Largo del Pozzo 71, 41125 Modena, Italy. Tel. +39.059.4224896. E-mail: irene.coloretti@gmail.com

Contributions: CI, BS, TM, GM, design of the study, analysis and interpretation of data and drafting the manuscript; BE, SV, EM, SM, DL, FR, MM, collection and analysis of data; CE, TR, MC, GG, CA, revising the manuscript critically for important intellectual content.

Conflict of interest: The authors declare that they have no competing interests, and all authors confirm accuracy.

Funding: The study was not supported by specific funds.

Availability of data and materials: The datasets used and/or analysed during the current study are available from the corresponding author on reasonable request.

Ethics approval and consent to participate: The study was approved by the Ethics Committee of Area Vasta Nord Emilia Romagna (n. 289/2020, 7 April 2020) who deemed informed consent unnecessary because of the retrospective design.

Consent for publication: Not applicable. 


\section{Background}

Since February $20^{\text {th }}, 2020$ Italy has been overwhelmed by SARS-CoV-2 virus outbreak and several patients with interstitial pneumonia and respiratory failure requiring mechanical ventilation were admitted to our intensive care unit (ICU) [1]. Unfortunately, so far there are no validated therapies to prevent or treat the severe acute respiratory distress syndrome (ARDS) caused by this novel virus and, thus, the case-fatality rate in patients admitted to ICU is extremely high, ranging from 30 to $80 \%$ [2-7]. Therefore, along with the maintenance of vital functions by supportive treatments, effective therapies in COVID-19 are urgently needed and several trials are underway worldwide for evaluating the effects of new and old antivirals agents, hydroxychloroquine, convalescent plasma, specific immunosuppressive agents and others [8-10].

In the last weeks, the scientific community provided a tremendous improvement in the knowledge of mechanisms involved in COVID-19 pathobiology. Uncontrolled immune response characterized by systemic hyper-inflammation with an abnormal increase of circulating cytokines and chemokines (the so-called cytokine storm) is common in critically ill COVID-19 patients and seems to have a pivotal role in lung tissue damage, increase in vascular permeability and clots formation [11]. To reduce the burden of this hyperinflammatory state many authors postulated the use of drugs with selective (e.g., anti-interleukin 1 and 6) and unselective (e.g., steroids, immunoglobulins) block of inflammatory mediators. The assessment of serum levels of proinflammatory biomarkers and cytokines has become common in clinical practice for early recognizing COVID-19 patients at risk for clinical worsening and who could benefit by the use of specific immunosuppressive agents [12]. On February $25^{\text {th }} 2020$, we admitted the first patient with severe COVID-19 pneumonia to our ICU. Fifteen days later, based on the above mentioned pathophysiological considerations and the high mortality rate of patients, without any specific treatment showing real efficacy in improving clinical conditions, the use of tocilizumab (a recombinant humanized monoclonal antibody directed against both the soluble interleukin- 6 receptor and the membrane-bound receptor) or anakinra (a recombinant interleukin-1 receptor antagonist), in case of shortage of intravenous tocilizumab, has been included in the local protocol for the management of COVID-19 as an optional treatment in critically ill patients with the need of non-invasive or invasive ventilatory support because of moderate to severe ARDS. Thereafter, on April $6^{\text {th }}$ the first experience on the use of tocilizumab was published, with the same rational in the attempt of the use of cytokine-blocking agents, despite providing insufficient level of evidence and not fully positive results [13].

This cohort study aimed to evaluate the effects of the therapy with tocilizumab or anakinra on in-hospital mortality in patients requiring mechanical ventilation for severe COVID-19 pneumonia and admitted to ICU. We hypothesized that the use of tocilizumab or anakinra would be safe and associated with in-hospital mortality rate reduction in the analysis adjusted for the major factors related to poor outcome.

\section{Methods}

\section{Design, population and protocol}

This retrospective cohort observational study included consecutive adult patients ( $\geq 18$ years) admitted to the ICU of University Hospital of Modena with ICU stay $>24$ hours, moderate to severe ARDS, requiring invasive or non-invasive mechanical ventilation and laboratory-confirmed SARS-CoV-2 infection from February $25^{\text {th }}$ to April $6^{\text {th }}$. Moderate to severe ARDS was defined as new or worsening respiratory failure with bilateral opacities and $\mathrm{PaO}_{2} / \mathrm{FiO}_{2} \leq 200 \mathrm{mmHg}$ with positive end-expiratory pressure $\geq 5$ $\mathrm{cmH}_{2} \mathrm{O}$ not fully explained by cardiac failure, fluid overload, pleural effusions and lobar or lung collapse [14]. SARS-CoV-2 infection was defined as a positive result of real-time reverse transcriptase-polymerase chain reaction (RT-PCR) assay of nasopharyngeal swabs or lower respiratory tract specimens. The study was approved by the Ethics Committee of Area Vasta Nord Emilia Romagna (n. 289/2020, 7 April 2020) who deemed informed consent unnecessary because of the retrospective design. The informed consent for off-label use of drugs is standard procedure in our Hospital with specific annotation on clinical charts. In unconscious patients, as for procedure, we communicate the offlabel use to relatives and annotate it on clinical charts.

All the patients received standard ICU monitoring and supportive care, including mechanical protective ventilation, as recommended by the WHO guidelines [15] and specific therapies according to national [16] and local protocol for COVID-19 treatment including hydroxychloroquine, azithromycin if suspicion of bacterial respiratory superinfection, low molecular weight heparin for prophylaxis of deep vein thrombosis according to individual bodyweight and renal function, and antiretroviral therapy with lopinavir/ritonavir or darunavir/cobicistat (removed on $22 \mathrm{March}$ ). The local protocol allowed the use of steroids (methylprednisolone $2 \mathrm{mg} / \mathrm{kg} /$ day) to prevent the onset of pulmonary fibrosis in patients who maintained a $\mathrm{PaO}_{2} / \mathrm{FiO}_{2}$ ratio $<150$ for at least 7 days of mechanical ventilation [17].

We compared the cohort of patients treated with cytokine-blocking agents (TOCI or ANA) with a control group composed by nontreated patients because admitted in ICU before the introduction of these cytokine-blocking therapies in our institutional protocol. Since March $5^{\text {th }}$, for the reasons described above (see Introduction), the local ICU management protocol included the option for off-label use of tocilizumab (TOCI) or anakinra (ANA), when intravenous TOCI was not available due to market shortage, in patients with moderate or severe ARDS and the need of mechanical ventilation (non-invasive or invasive). Patients with coexistent infection other than COVID-19, chronic use of steroids or other immunosuppressive agents, neutrophils $<500 / \mathrm{mmc}$ or platelets $<50.000 / \mathrm{mmc}$, gastrointestinal tract condition that might predispose to bowel perforation and severe hematologic, renal or liver impairment were excluded from this therapeutic option. Nevertheless, the decision for therapy with TOCI or ANA was left to the discretion of the treating team considering the benefit to risk ratio in the individual patient. TOCI was administered intravenously at the dosage of $8 \mathrm{mg} / \mathrm{kg}$ of body weight (up to a maximum dosage of $800 \mathrm{mg}$ per infusion) in two doses, 12 hours apart. ANA was used intravenously at the dose of $400 \mathrm{mg} / \mathrm{die}$ for 14 days or until the patient was weaned from mechanical ventilation or adverse events related to therapy occurred. The TOCI and ANA schedules were based on dosages indicated for the treatment of cytokine release syndrome [18] and macrophage activated syndrome $[19,20]$. The standard supportive management in ICU did not change during the study period.

\section{Data collection and analysis}

Demographics, co-morbidities, medications, and laboratory values were collected by reviewing electronic medical records. The primary endpoint was in-hospital survival after ICU admission. Secondary endpoints were ICU mortality, ICU-free days censored at day 30, invasive and non-invasive ventilator-free days (VFDs) at day 30 after ICU admission, the incidence of secondary infections within hospital stay. All enrolled patients achieved the follow up period. The association between immunotherapy and in- 
hospital mortality was estimated by multivariable Cox proportional hazards regression model including immunotherapy, variables with $\mathrm{p}<0.2$ at unadjusted analysis (age, co-morbidities, SAPS II and invasive ventilation at ICU admission).

To evaluate the independent association of TOCI and ANA with mortality, a second multivariable Cox proportional hazards regression model was built including the previous covariates with stratification of immunotherapies in the two different treatment arms. To further reduce the effects of confounding variables we performed a secondary analysis by using propensity score for patients matching. The individual propensity to be treated with TOCI or ANA was estimated by a multivariable logistic regression model that included the same covariates as the Cox regression; the nearest-neighbour method was applied to the propensity-score matching analysis. An additional sensitivity analysis included the same set of analyses was performed only in the population with invasive mechanical ventilation within 2 hours after ICU admission. Non-parametric and $\chi-2$ tests were used as appropriate for the unadjusted comparison between controls and treated patients of demographic and baseline values, and outcomes. Cumulative survival analysis censored at day 90 was performed using the KaplanMeier method and the log-rank test was then used to examine differences in the curves between the groups. Patients discharged from the hospital before day 90 were considered survived. All tests were two-tailed with $\mathrm{p}<0.05$ considered significant. SPSS version 22.0 package (SPSS Inc., Chicago, IL, USA) was used to perform statistical analysis.

\section{Results}

In the study period, ninety-nine COVID-19 patients were admitted to our ICU, of whom 94 met the inclusion criteria. Sixty- six patients $(70 \%)$ received selective cytokine blockade therapy (immunotherapy group), 49 (52\%) with 2 doses of TOCI and 17 $(18 \%)$ with ANA for a median of 8 days (IQR 7-11). In 26 patients (53\%) the first dose of TOCI was administered before ICU admission (median time 24 -IQR 12-48 hours) and in 23 (43\%) after ICU admission (median time 6 -IQR 0-24 hours). ANA was started after ICU admission in all the patients (median time 4-IQR 0-24 hours) and was stopped in $9(53 \%)$ patients for weaning from mechanical ventilation, in $4(24 \%)$ for death during treatment and $3(23 \%)$ patients completed the full therapy course.

Demographic and clinical characteristics at ICU admission were similar in patients who received and who did not receive selective cytokine blockade therapy with exception of percentage of patients undergoing invasive ventilation within 2 hours from admission $(\mathrm{p}=0.006)$ and $\mathrm{PaO}_{2} / \mathrm{FiO}_{2}(\mathrm{p}=0.029)$ that were larger in control patients, and interleukin 6 blood concentration that was higher $(\mathrm{p}=0.019)$ in immunotherapy group (Table 1$)$. Twenty-four out of the 44 patients in non-invasive ventilation within 2 hours after ICU admission were subsequently intubated during ICU stay ( 3 out of 7 in the control group (43\%) and 21 out of $37(57 \%)$ in immunotherapy group, $\mathrm{p}=0.48)$. Fifty-six patients $[14$ in the control group and 43 in the immunotherapy group $(\mathrm{p}=0.17)]$ received steroids during ICU stay for a median of 5 days (IQR 5-7).

Crude analysis showed that selective cytokine blockade therapy provided $20 \%$ absolute risk reduction of in-hospital mortality compared to controls (OR 0.77, 95\% CI 0.56-1.05, $\mathrm{p}=0.069$ ) (Table 2). The Kaplan Meyer analysis for cumulative in-hospital survival (Figure 1) shows a significative effect of immunotherapy as a protective strategy $(\mathrm{p}=0.036)$. Cox regression multivariable analysis indicated that age, SAPS II, and immunotherapy were related to mortality risk, with an improvement in survival by the use of TOCI or ANA (HR 0.40, 95\% CI 0.19-0.83, p=0.015) (Table 3). The main cause of death in both groups was a multiorgan fail-

Table 1. Demographic and characteristics of the study populations.

\begin{tabular}{|c|c|c|c|c|}
\hline & All patients ( $\mathrm{n}=94)$ & Control $(n=28)$ & Immunotherapy (n=66) & p \\
\hline Age (median, IQR) & $63(56-70)$ & $62(55-68)$ & $63(57-71)$ & 0.297 \\
\hline Sex, male (n, \%) & $75(79.8)$ & $22(78.6)$ & $53(80.3)$ & 0.848 \\
\hline Comorbidities (n, \%) & $67(71.3)$ & $22(78.6)$ & $45(68.2)$ & 0.309 \\
\hline Hypertension & $47(50)$ & $14(50)$ & $33(50)$ & \\
\hline Body mass index $>30$ & $14(14.9)$ & $4(14.3)$ & $10(15.2)$ & \\
\hline Hyperlipidaemia & $6(6.4)$ & $2(7.1)$ & $4(6.1)$ & \\
\hline Diabetes & $15(15.9)$ & $6(21.4)$ & $9(13.6)$ & \\
\hline Any other & $27(28.7)$ & $6(21.4)$ & $21(31.8)$ & \\
\hline SOFA (median, IQR) & $4(3-5)$ & $4(3-6)$ & $4(3-5)$ & 0.849 \\
\hline SAPS II (median, IQR) & $31.5(27-37)$ & $30(25-38)$ & $32(27-37)$ & 0.646 \\
\hline Ventilation at ICU admission (n, \%) & & & & 0.006 \\
\hline Invasive* & $50(52.3)$ & $21(75)$ & $29(43.9)$ & \\
\hline Non-invasive & $44(46.8)$ & $7(25)$ & $37(56.1)$ & \\
\hline $\mathrm{PaO}_{2} / \mathrm{FiO}_{2}$ (mmHg, median, IQR) & $100(80-123)$ & $113(86-161)$ & 96 (74-117) & 0.029 \\
\hline D-dimer (ng/ml; median, IQR) & $1985(870-3700)$ & $1915(750-2660)$ & $2085(940-8650)$ & 0.110 \\
\hline LDH (U/L; median, IQR) & $762(611-1031)$ & $700.5(588-1039.5)$ & $774(655-1031)$ & 0.549 \\
\hline CRP (mg/L; median, IQR) & $15.4(6.2-22.7)$ & $16.3(7.5-26.2)$ & $15.3(6.1-21.1)$ & 0.359 \\
\hline PCT (ng, ml; median, IQR) & $0.3(0.1-1.1)$ & $0.4(0.2-0.8)$ & $0.3(0.1-1.2)$ & 0.351 \\
\hline IL6 (pg/ml; median, IQR) ${ }^{\circ}$ & $452.2(207.2-1483.3)$ & $242.9(115.5-386)$ & $541.6(214.5-1526)$ & 0.019 \\
\hline Lymphocyte count (cells/mcl; median, IQR) & $0.64(0.420 .89)$ & $0.56(0.390 .85)$ & $0.66(0.46-0.91)$ & 0.215 \\
\hline
\end{tabular}

SOFA, simplified organ failure assessment; SAPS, simplified acute physiological score II, $\mathrm{PaO}_{2}$, arterial partial pressure of oxygen; FiO $\mathrm{O}_{2}$, fraction of oxygen in inspired mixture; LDH, Lactate dehydrogenase; CRP, C-reactive protein; PCT, procalcitonin; IL6, interleukin 6; * within 2 hours after ICU admission; ${ }^{\circ}$ measured in 65 patients, 10 controls, 55 Immunotherapy. 
ure due to persistent and intractable hypoxemia $(n=15,75 \%$ in the Immunotherapy group and $n=11,79 \%$ in the controls). Baseline and mortality rate at day 30 were similar in patients treated with ANA and treated with TOCI. The Cox regression model with TOCI and ANA as separate covariates indicated that TOCI decreased (HR $0.31-95 \%$ CI 0.13-0.73, p=0.007) and ANA showed a trend in decreasing the risk of mortality (HR 0.46 - 95\% CI 0.17-1.26, $\mathrm{p}=0.130$ ). The multivariable analysis including 11 controls and 55 immunotherapy patients matched by propensity-score (well-balanced for all covariates) also indicated that the use of interleukin selective blockade agents is associated with reduced risk of mortality (HR 0.23- 95\% CI 0.09-0.56, $\mathrm{p}=0.001$ ).

The invasive ventilation free days $(p=0.035)$, total ventilation (invasive and non-invasive) free days $(\mathrm{p}=0 ., 132)$ as well as ICU free days $(\mathrm{p}=0.07)$ at day-30 were larger in immunotherapy group. The rate of patients with new infections was similar in the 2 groups; the infections were sustained mostly by Gram-positive bacteria $(65.8 \%)$ with respiratory tract $(76.3 \%)$ and bloodstream $(28.9 \%)$ as main sites of infection. As for viral infections, 4 patients in the control group and 5 in the immunotherapy group were treated by ganciclovir for cytomegalovirus reactivation; 1 patient who received ANA and 4 patients who received TOCI were treated with acyclovir for herpes simplex virus type 1 reactivation. No differences in secondary outcomes were observed between patients treated with ANA or TOCI. The sensitivity analysis in patients with invasive ventilation at ICU admission (21 patients in TOCI, 8 in ANA and 21 in controls) confirmed the potential association between selective cytokine blockade therapy and the reduced risk of in-hospital mortality.

\section{Discussion}

The results of this cohort observational study indicate that selective cytokine blockade with tocilizumab or anakinra in critically ill patients with moderate to severe ARDS pneumonia and need of mechanical ventilation seems to be well tolerated and to

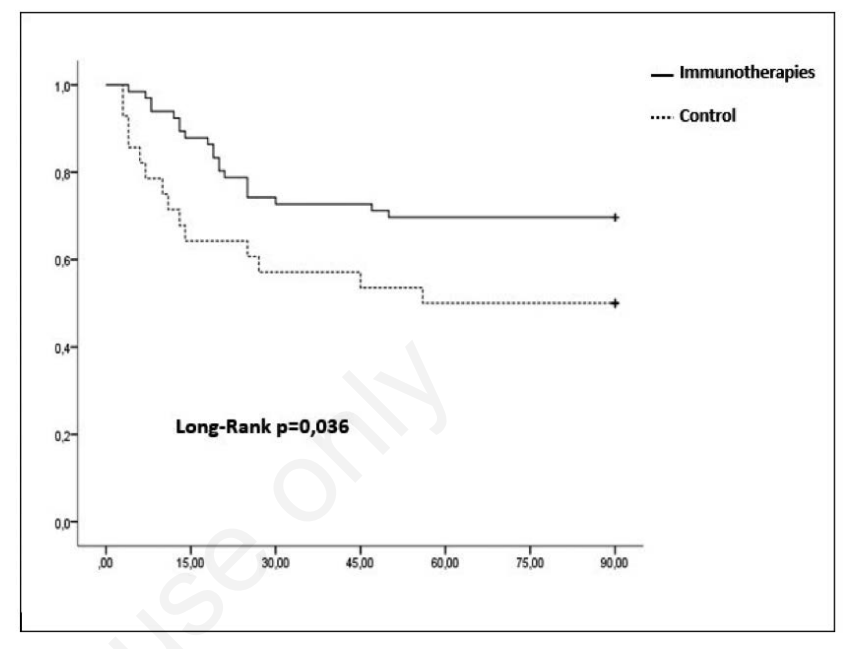

Figure 1. Kaplan Meyer Curves for survival probability at day 30.

Table 2. Main outcomes in all population, controls and treated patients.

\begin{tabular}{|c|c|c|c|c|}
\hline & All patients $(\mathrm{n}=94)$ & Control $(n=28)$ & Immunotherapy ( $\mathrm{n}=66)$ & $\mathbf{p}$ \\
\hline Hospital mortality (n, \%) & $34(36.2)$ & $14(50.0)$ & $20(30.3)$ & 0.069 \\
\hline 30-day mortality (n, \%) & $29(30.9)$ & $12(42.9)$ & $17(25.8)$ & 0.101 \\
\hline ICU mortality (n, \%) & $27(28.7)$ & $11(39.3)$ & $16(24.2)$ & 0.140 \\
\hline ICU-free days at 30 day (median, IQR) & $16.5(0-25)$ & $0(0-22)$ & $18.5(0-26)$ & 0.072 \\
\hline Invasive ventilation free days at 30-day (median, IQR) & $20.5(0-28)$ & $0(0-26)$ & $22.5(0-29)$ & 0.035 \\
\hline Ventilation-free days at 30-day (median, IQR) & $16.5(0-25)$ & $0(0-23.5)$ & $18.5(0-26)$ & 0.132 \\
\hline Patients with new bacterial infections during hospital stay (n, \%) & $38(40.4)$ & $11(39.3)$ & $27(40.9)$ & 0.883 \\
\hline
\end{tabular}

Table 3. Odds ratios and confidence interval obtained by unadjusted univariate and adjusted Cox regression analysis for in-hospital mortality. Data for survived and not survived during hospital stay are also reported.

\begin{tabular}{|c|c|c|c|c|}
\hline & $\begin{array}{l}\text { Survived } \\
(\mathrm{n}=60)\end{array}$ & $\begin{array}{l}\text { Not survived } \\
(n=34)\end{array}$ & $\begin{array}{c}\text { Unadjusted OR (95\% CI); } \\
\text { p }\end{array}$ & $\begin{array}{c}\text { Adjusted HR (95\% CI); } \\
\text { p }\end{array}$ \\
\hline Age (median; IQR) & $61(54-67)$ & $69(62-75)$ & $\begin{array}{c}1.6(1.01-1.11) \\
0.018\end{array}$ & $\begin{array}{c}1.07(1.02-1.12) \\
0.010\end{array}$ \\
\hline Comorbidities (n, \%) & $38(63.3)$ & $29(85.3)$ & $\begin{array}{c}1.44(1.09-1.89) \\
0.019\end{array}$ & $\begin{array}{c}1.33(0.51-3.51) \\
0.559\end{array}$ \\
\hline SAPS II score (median; IQR) & $27(24.5-32)$ & $36(34-43)$ & $\begin{array}{c}1.07(1.03-1.11) \\
0.001\end{array}$ & $\begin{array}{c}1.05(1.01-1.10) \\
0.011\end{array}$ \\
\hline Invasive ventilation at ICU admission* (n, \%) & $23(38.3)$ & $27(79.4)$ & $\begin{array}{c}1.83(1.32-2.53) \\
<0.001\end{array}$ & $\begin{array}{c}2.38(0.97-5.86) \\
0.059\end{array}$ \\
\hline Immunotherapy (n, \%) & $46(76.7)$ & $20(58.8)$ & $\begin{array}{c}0.72(0.48-1.07) \\
0.058\end{array}$ & $\begin{array}{c}0.40(0.19-0.83) \\
0.015\end{array}$ \\
\hline
\end{tabular}


improve in-hospital survival rate. Based on recent reports [3,21], the pathobiology of interstitial pneumonia and acute respiratory failure by SARS-CoV-2 infection relies on the predominant role of hyper-inflammation in the context of a complex dysregulated immune function [22]. As observed by others [3,23], our patients showed a pronounced pro-inflammatory state at ICU admission with elevated serum CRP, D-Dimer, LDH and IL6 levels associated with lymphopenia. Subsequently to the poor outcome observed in the first reports on COVID-19 patients requiring ICU admission, the attractive hypothesis of controlling the exaggerated cytokine response by using different immunomodulating agents has been early proposed [24] and many randomized trials are ongoing.

TOCI and ANA, among immunomodulatory drugs, represent two treatment options in this context. TOCI, a selective interleukin-6 receptor antagonist, was approved for the treatment of autoimmune diseases and chimeric antigen receptor T-cell therapyinduced cytokine release syndrome [25]. Early reporting of clinical experiences with TOCI in COVID-19 patients have been published at the end of July 2020. The most of reported experiences deal with patients before ICU admission and/or mechanical ventilation [26]. As refers to ICU patients, in a retrospective analysis of 25 unmatched ICU patients, TOCI at the median dose of $5,7 \mathrm{mg} / \mathrm{kg}$ showed a significant reduction in invasive mechanical ventilation at day 14 , although $90 \%$ of patients showed adverse events [27]. In a single-centre study including 100 unmatched COVID-19 patients with mechanical ventilation, the authors observed an improvement of the respiratory severity using a disease-specific scale and the decreasing of laboratory inflammation parameters after two intravenous administrations of $8 \mathrm{mg} / \mathrm{kg}$ of TOCI [28]. Two recent experiences, according with our results, report improvement in clinical outcomes of ICU patients affected by COVID-19 receiving tocilizumab [29,30]. In particular, one of these studies reports a decreased hospital mortality in treated patients, with similar associations in higher-severity subgroups [30].

As refers to ANA, it is a recombinant IL-1 receptor antagonist administered to treat autoinflammatory disorders and recently used in post-myocardial infarction remodelling and diabetes [31]. In the context of COVID-19 acute respiratory distress syndrome, a retrospective study was conducted on 29 patients treated with highdosage ANA compared to 16 control ones; patients were non-invasively ventilated outside of the ICU. Compared with standard treatment, high-dose ANA was associated with a higher survival rate at 21 days and with a reduction in C-reactive protein and with progressive improvement in $\mathrm{PaO}_{2} / \mathrm{FiO}_{2}$. However, high-dose treatment was discontinued for adverse events in $24 \%$ of the treated patients [32].

To our knowledge, our study is the first to have evaluated the effects of the selective cytokine blockade on in-hospital mortality in ICU admitted patients requiring mechanical ventilation for ARDS due to COVID-19. Beyond the significant risk reduction compared to our controls, the in-hospital mortality $(36.2 \%$ in all the patients and $54 \%$ in those with invasive ventilation at ICU admission) observed in our patients treated with selective cytokine blockade was also lower than mortality observed in other reports, even when only patients underwent invasive ventilation are considered. A large case series of ICU patients coming from Wuhan, China, showed an overall mortality rate at day 28 of $39 \%$ but increased to $97 \%$ in patients with invasive mechanical ventilation. Another retrospective study coming from Wuhan enrolled 52 critically ill ICU patients with a 28-day mortality rate of $61.5 \%$ [6]. In the Italian Lombardy ICU Network series, the hospital mortality rate of 1,715 patients admitted to ICU was 50.3\% [33] and among 5,700 hospitalized patients in the New York Area, 373 patients needed ICU care with an ICU mortality rate of 78\% [34]. Finally, the ICNARC report on COVID-19 updated on $1^{\text {st }}$ May showed an ICU mortality of $62 \%$ in
3,508 patients with advanced respiratory support [35]. Remarkably, following previous reports, we did not observe any severe adverse event attributable with certainty to the use of TOCI or ANA [36]. The rate of secondary infections, mostly ventilator acquired pneumonia and catheter-related bloodstream infections by Gram-positive microorganisms, was high compared to non-COVID-19 patients admitted to our ICU (internal data from Prosafe-Giviti project https://giviti.marionegri.it), but the use of cytokine selective blockade therapy did not increase the risk in comparison with controls treated in our centre. Nevertheless, in our cohort, the incidence of adverse events by ANA and TOCI could have been underestimated because of frequent clinical and laboratory alterations occurring in critically ill patients with severe ARDS or other organ dysfunctions. In fact, in 5 patients treated with cytokine blocking agents and long ICU stay we observed late reactivation of herpes simplex virus type 1 and, besides, two COVID-19 patients treated with TOCI in other hospitals were transferred to our ICU for acute liver failure, without ARDS, due to herpes simplex virus type 1 reactivation (submitted for publication).

Our study had several limitations that are mainly due to the observational design and to the small size of the cohort studied. Although the use of propensity score for adjusting the multivariable analysis and for patients matching is considered an effective method in non-randomized trials, it is possible that some amount of unmeasured confounding factors still remains. Nevertheless, it is noteworthy the all the data analysis (unadjusted, adjusted without and with propensity score) pointed out the same signal with a potential benefit in terms of survival by using selective cytokine blockade. The change of intubation rules during the study period with more permissive use of non-invasive ventilation in patients with severe ARDS could have introduced a bias. However, the sensitivity analysis in patients with invasive ventilation at ICU admission (within 2 hours) confirmed the reduction in mortality by using TOCI and ANA (Table 3). Of note, evaluating together the results of patients treated with two different drugs represents a limitation of our study. Moreover, the small number of patients treated with ANA and the different timing of administration of the two drugs hinder more robust analysis on the potential different effect of TOCI and ANA on patient survival, even if Cox multivariable regression seems to indicate a greater effect when using TOCI.

\section{Conclusions}

In conclusion, our experience indicated that tocilizumab and anakinra appear to be safe and to improve survival in patients with moderate to severe ARDS by SARS-CoV-2 infection. The use of drugs able to provide a selective cytokine blockade may represent a promising therapeutic option in the treatment of mechanically ventilated COVID-19 patients even though, given our study's limitations, our results need confirmatory results from high-quality randomized controlled trials.

\section{Acknowledgements}

The manuscript has been released as a pre-print at ResearchSquare platform (https://www.researchsquare.com/article/rs-37561/v2). We are more than grateful to nurses and associated healthcare personnel of the Modena University Hospital for the unbelievable and restless activity during the COVID-19 emergency. A special word of thanks to the Medical Direction, Clinical Engineering, and Information Technology Departments of the Hospital for the tremendous effort in organization and support of clinical activities. 


\begin{tabular}{ll}
\hline Abbreviations \\
ANA: & anakinra; \\
ARDS: & acute respiratory distress syndrome; \\
$\mathrm{COVID}-19:$ & coronavirus disease-19; \\
$\mathrm{CRP}:$ & C-reactive protein; \\
$\mathrm{FiO}_{2}:$ & fraction of oxygen in inspired mixture \\
$\mathrm{ICU}^{2}$ & intensive care unit; \\
$\mathrm{IL6}:$ & interleukin-6; \\
$\mathrm{LDH}:$ & lactate dehydrogenase; \\
$\mathrm{PaO}:$ & arterial partial pressure of oxygen; \\
PCT: & procalcitonin; \\
TOCI: & tocilizumab; \\
SOFA: & simplified organ failure assessment; \\
SAPS II: & implified acute physiological score II.
\end{tabular}

References

1. Grasselli G, Zangrillo A, Zanella A, Antonelli M, Cabrini L, Castelli A, et al. Baseline characteristics and outcomes of 1591 patients infected with SARS-CoV-2 admitted to ICUs of the Lombardy Region, Italy. JAMA 2020;323:1574-81.

2. Wu C, Chen X, Cai Y, Xia J, Zhou X, Xu S, et al. Risk factors associated with acute respiratory distress syndrome and death in patients with coronavirus disease 2019 pneumonia in Wuhan, China. JAMA Intern Med 2020;180:934-43.

3. Zhou F, Yu T, Du R, Fan G, Liu Y, Liu Z, et al. Clinical course and risk factors for mortality of adult inpatients with COVID19 in Wuhan, China: a retrospective cohort study. Lancet 2020;395:1054- 62 .

4. Bhatraju PK, Ghassemieh BJ, Nichols M, Kim R, Jerome KR, Nalla AK, et al. Covid-19 in critically ill patients in the Seattle Region - Case series. N Engl J Med 2020;382:2012-2.

5. Huang C, Wang Y, Li X, Ren L, Zhao J, Hu Y, et al. Clinical features of patients infected with 2019 novel coronavirus in Wuhan, China. Lance. 2020;395:497-506.

6. Yang X, Yu Y, Xu J, Shu H, Xia J, Liu H, et al. Clinical course and outcomes of critically ill patients with SARS-CoV-2 pneumonia in Wuhan, China: a single-centered, retrospective, observational study. Lancet Respir Med 2020;8:475-81.

7. Arentz M, Yim E, Klaff L, Lokhandwala S, Riedo FX, Chong $\mathrm{M}$, et al. Characteristics and outcomes of 21 critically ill patients with COVID-19 in Washington State. JAMA 2020;323:1612-4.

8. Pascarella G, Strumia A, Piliego C, Bruno F, Del Buono R, Costa F, et al. COVID-19 diagnosis and management: a comprehensive review. J Intern Med 2020;288:192-206.

9. Wang Y, Zhang D, Du G, Du R, Zhao J, Jin Y, et al. Remdesivir in adults with severe COVID-19: a randomised, double-blind, placebo-controlled, multicentre trial. Lancet 2020;395:156978.

10. RECOVERY Collaborative Group, Horby P, Lim WS, Emberson JR, Mafham M, Bell JL, et al. Dexamethasone in hospitalized patients with Covid-19 - Preliminary report. N Engl J Med 2020;384:693-704.

11. Jose RJ, Manuel A. COVID-19 cytokine storm: the interplay between inflammation and coagulation. Lancet Respir Med 2020;8:e46-7.

12. Ye Q, Wang B, Mao J. The pathogenesis and treatment of the 'Cytokine Storm' in COVID-19. J Infect 2020;80:607-13.

13. Luo P, Liu Y, Qiu L, Liu X, Liu D, Li J. Tocilizumab treatment in COVID-19: A single center experience. J Med Virol 2020;92:814-8.
14. ARDS Definition Task Force, Ranieri VM, Rubenfeld GD, Thompson BT, Ferguson ND, Caldwell E, et al. Acute respiratory distress syndrome: the Berlin Definition. JAMA 2012;307:2526-33.

15. World Health Organization. Clinical management of severe acute respiratory infection (SARI) when COVID-19 disease is suspected: interim guidance, 13 March 2020. Available from: https://apps.who.int/iris/handle/10665/331446

16. National Society of Infectious Diseases' (SIMIT) Guidelines for the management of COVID-19 infection. Accessed on: April 5th 2020. Available from: http://www.simit.org/medias/ 1569-covid19-vademecum-13-03-202.pdf

17. Steinberg KP, Hudson LD, Goodman RB, Hough CL, Lanken PN, Hyzy R, et al. Efficacy and safety of corticosteroids for persistent acute respiratory distress syndrome. N Engl J Med 2006;354:1671-84.

18. Le RQ, Li L, Yuan W, Shord SS, Nie L, Habtemariam BA, et al. FDA approval summary: Tocilizumab for treatment of chimeric antigen receptor $\mathrm{T}$ cell-induced severe or life-threatening cytokine release syndrome. Oncologist 2018;23:943-7.

19. Mehta P, Cron RQ, Hartwell J, Manson JJ, Tattersall RS. Silencing the cytokine storm: the use of intravenous anakinra in haemophagocytic lymphohistiocytosis or macrophage activation syndrome. Lancet Rheumatol 2020;2:E358-67.

20. Futami M, Suzuki K, Kato S, Ohmae S, Tahara Y, Nojima M, et al. The novel multi-cytokine inhibitor TO-207 specifically inhibits pro-inflammatory cytokine secretion in monocytes without affecting the killing ability of CAR T cells. PLoS One 2020;15:e0231896.

21. Ruan Q, Yang K, Wang W, Jiang L, Song J. Clinical predictors of mortality due to COVID-19 based on an analysis of data of 150 patients from Wuhan, China. Intensive Care Med 2020;46:846-8.

22. Giamarellos-Bourboulis EJ, Netea MG, Rovina N, Akinosoglou K, Antoniadou A, Antonakos N, et al. Complex immune dysregulation in COVID-19 patients with severe respiratory failure. Cell Host Microbe 2020;27:992-1000.e3.

23. Lippi G, Plebani M. Laboratory abnormalities in patients with COVID-2019 infection. Clin Chem Lab Med 2020;58:1131-4.

24. Mehta P, McAuley DF, Brown M, Sanchez E, Tattersall RS, Manson JJ, et al. COVID-19: consider cytokine storm syndromes and immunosuppression. Lancet 2020;395:1033-4.

25. Rubbert-Roth A, Furst DE, Nebesky JM, Jin A, Berber E. A Review of recent advances using tocilizumab in the treatment of rheumatic diseases. Rheumatol Ther 2018;5:21-42.

26. Guaraldi G, Meschiari M, Cozzi-Lepri A, Milic J, Tonelli R, Menozzi M, et al. Tocilizumab in patients with severe COVID19: a retrospective cohort study. Lancet Rheumatol 2020;2:e474-84.

27. Alattar R, Ibrahim TBH, Shaar SH, Abdalla S, Shukri K, Daghfal JN, et al. Tocilizumab for the treatment of severe coronavirus disease 2019. J Med Virol 2020;92:2042-9.

28. Knorr JP, Colomy V, Mauriello CM, Ha S. Tocilizumab in patients with severe COVID-19: A single-center observational analysis. J Med Virol 2020;92:2813-20.

29. Eimer J, Vesterbacka J, Svensson AK, Stojanovic B, Wagrell C, Sönnerborg A, et al. Tocilizumab shortens time on mechanical ventilation and length of hospital stay in patients with severe COVID19: a retrospective cohort study. J Intern Med 2021;289:434-6.

30. Biran N, Ip A, Ahn J, Go RC, Wang S, Mathura S, et al. Tocilizumab among patients with COVID19 in the intensive care unit: a multicentre observational study. Lancet Rheumatol 2020;2:e603-12.

31. Dinarello CA, van der Meer JWM. Treating inflammation by blocking interleukin-1 in humans. Semin Immunol 2013;25: 
469-84.

32. Cavalli G, De Luca G, Campochiaro C, Della-Torre E, Ripa M, Canetti D, et al. Interleukin-1 blockade with high-dose anakinra in patients with COVID-19, acute respiratory distress syndrome, and hyperinflammation: a retrospective cohort study. Lancet Rheumatol 2020;2:E325-31.

33. Grasselli G, Greco M, Zanella A, Albano G, Antonelli M, Bellani $\mathrm{G}$, et al. Risk factors associated with mortality among patients with COVID-19 in intensive care units in Lombardy, Italy. JAMA Intern Med 2020;180:1345-55.

34. Richardson S, Hirsch JS, Narasimhan M, Crawford JM, McGinn T, Davidson KW, et al. Presenting characteristics, comorbidities, and outcomes among 5700 patients hospitalized with COVID-19 in the New York City area. JAMA 2020;323:2052-9.

35. ICNARC report on COVID-19 in critical care. 01 May 2020. Available from: https://www.icnarc.org/DataServices/ Attachments/Download/f48efee2-d38b-ea11-912500505601089b

36. Sciascia S, Aprà F, Baffa A, Baldovino S, Boaro D, Boero R, et al. Pilot prospective open, singlearm multicentre study on off-label use of tocilizumab in patients with severe COVID-19. Clin Exp Rheumatol 2020;38:529-32.

\section{*Modena COVID-19 Working Group (MoCo19):}

Intensive Care Unit: Massimo Girardis, Alberto Andreotti, Emanuela Biagioni, Filippo Bondi, Stefano Busani, Giovanni Chierego, Marzia Scotti, Lucia Serio, Annamaria Ghirardini, Marco Sita, Stefano De Julis, Lara Donno, Lorenzo Dall'Ara, Fabrizio Di Salvo, Carlotta Farinelli, Laura Rinaldi, Ilaria Cavazzuti, Andrea Ghidoni, Antonio Buono, Elena Ferrari, Daniela Iseppi, Anna Maria Ardito, Irene Coloretti, Sophie Venturelli, Elena Munari, Martina Tosi, Erika Roat, Ilenia Gatto, Marco Sarti.

Immuno-Lab: Andrea Cossarizza, Caterina Bellinazzi, Rebecca Borella, Sara De Biasi, Anna De Gaetano, Lucia Fidanza, Lara Gibellini, Anna Iannone, Domenico Lo Tartaro, Marco Mattioli, Milena Nasi, Annamaria Paolini, Marcello Pinti.

Infectious Disease Unit: Cristina Mussini, Giovanni Guaraldi, Marianna Meschiari, Alessandro Cozzi-Lepri, Jovana Milic, Marianna Menozzi, Erica Franceschini, Gianluca Cuomo, Gabriella Orlando, Vanni Borghi, Antonella Santoro, Margherita Di Gaetano, Cinzia Puzzolante, Federica Carli, Andrea Bedini, Luca Corradi. Respiratory Diseases Unit: Enrico Clini, Roberto Tonelli, Riccardo Fantini, Ivana Castaniere, Luca Tabbì, Giulia Bruzzi, Chiara Nani, Fabiana Trentacosti, Pierluigi Donatelli, Maria Rosaria Pellegrino, Linda Manicardi, Antonio Moretti, Morgana Vermi, Caterina Cerbone.

Virology and Molecular Microbiology Unit: Monica Pecorari, William Gennari, Antonella Grottola, Giulia Fregni Serpini. 